\title{
Regulation of Contractile Proteins by Phosphorylation
}

\author{
Robert S. Adelstein, Laboratory of Molecular Cardiology, \\ National Heart, Lung, and Blood Institute, National Institutes of Health, \\ Bethesda, Maryland 20205
}

The two major contractile proteins, actin and myosin, are not only responsible for the contraction of skeletal, cardiac, and smooth muscles, but they also play an important role in the movement and changes in shape of vertebrate nonmuscle cells, such as leukocytes, liver cells, and platelets $(1,2)$. Practically all eukaryotic cells contain the two major contractile proteins actin and myosin. Understanding how these contractile proteins are regulated is important not only for insight into how the heart and skeletal muscles work, but also for understanding the role of smooth muscle cells in processes such as blood pressure and flow, food propulsion, airway constriction, and uterine contraction. In addition, the contractile proteins appear to play a part in controlling basic cellular functions, such as cell migration, cytokinesis, phagocytosis, and secretion. The ubiquity of actin and myosin and their role in regulating cellular functions make them of particular interest to a wide body of scientists trying to understand the basic physiology as well as the clinical pathology of muscle and nonmuscle cells.

Contractile proteins function by forming filaments. The filaments formed by actin are composed of two linear polymers of a globular protein $(42,000 \mathrm{~mol} \mathrm{wt})$, wrapped in the form of a helix. These filaments are unipolar and are attached to the cell membrane in nonmuscle cells, dense bodies in smooth muscle cells, and to the well-defined $\mathrm{Z}$ line structure in skeletal and cardiac muscle. Because of their size relative to myosin filaments they are referred to as "thin" filaments in muscle cells. Myosin filaments are bipolar and composed of large molecules $(460,000 \mathrm{~mol} \mathrm{wt})$, which are markedly asymmetric. Whereas actin is composed of a single

Dr. Adelstein's present address is Department of Cellular Biochemistry. The Hebrew University, Hadassah Medical School, Jerusalem, Israel.

Received for publication 24 August 1983. polypeptide chain, myosin is a hexamer composed of a pair of heavy chains $(200,000 \mathrm{~mol} \mathrm{wt})$ and two pairs of light chains (molecular weight ranging from 15,000 to 28,000 ) (Fig. 1). At one end the myosin molecule is globular in shape and it is this end that binds to actin and possesses an enzymatic site that hydrolyzes ATP. The tail portion of the myosin molecule is responsible for its assembly into bipolar filaments. Because of their diameter relative to actin, myosin filaments are referred to as "thick" filaments, in striated muscle and smooth muscle. In nonmuscle cells, on the other hand, where their existence as filaments may only be transitory, they have been difficult to observe.

Contractile activity results from the sliding of the myosin and actin filaments passed each other $(3,4)$. This sliding is brought about by the attachment of the globular heads of the myosin molecules to actin, a change in the conformation of the myosin head with respect to actin, and detachment of the myosin head followed by the subsequent reattachment at another site further down the actin filament. The energy necessary for the physical movement of the actin filaments passed the myosin filaments is supplied by ATP, and it is the ability of myosin to hydrolyze ATP under the stimulus of actin (the actin-activated ATPase activity of myosin) that allows for this chemical $\rightarrow$ mechanical transformation.

There is a fundamental difference between myosins isolated from nonmuscle and smooth muscle cells and those isolated from striated (i.e., skeletal and cardiac) muscles. This difference is not reflected in many of myosin's obvious physical and chemical properties, since all myosins resemble each other in most of these parameters. The difference concerns the manner in which myosin's ability to hydrolize ATP is regulated. In skeletal and cardiac muscle, regulation of the actinmyosin interaction occurs at the level of the actin filaments. Attached to each of these filaments is a complex of proteins, the troponins and tropomyosin, which 


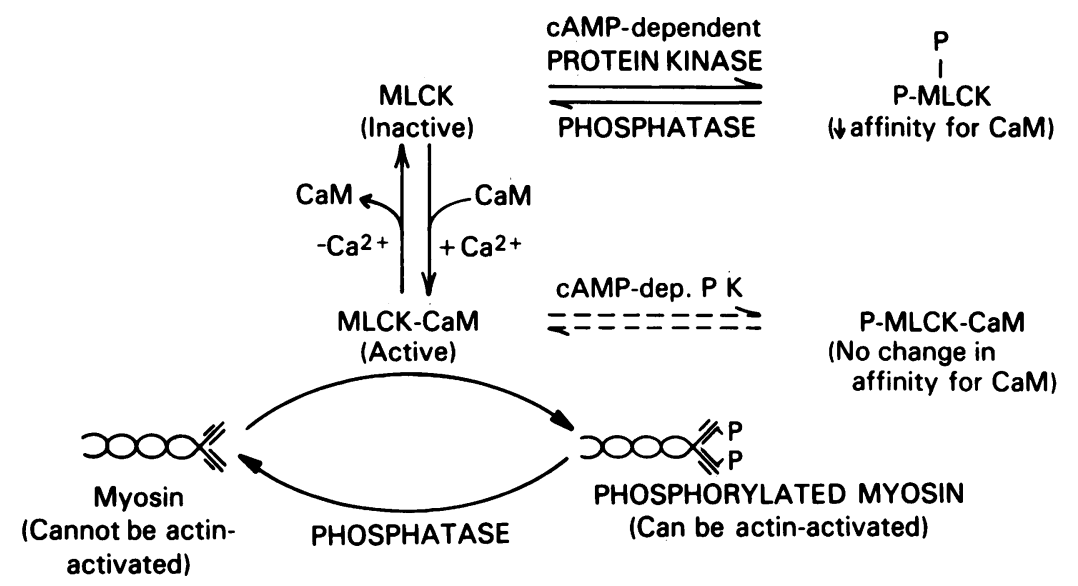

Figure 1 Diagram showing the effects of phosphorylating the myosin hexamer. Phosphorylation is catalyzed by a complex of MLCK and calcium-calmodulin (MLCK-CaM). MLCK is itself a substrate for cAMP-dependent protein kinase. The rate and stoichiometry of MLCK phosphorylation depends on whether CaM is bound to MLCK.

play the major role in regulating the actin-activated Mg ATPase activity of myosin. Contraction of skeletal and cardiac muscle is initiated when the calcium concentration inside these cells increases from $\sim 10^{-7}$ to $10^{-5} \mathrm{M}$. Calcium then binds to one component of the troponin complex, troponin $\mathrm{C}$, and this initiates a series of conformational changes allowing for the contraction of the actin and myosin filaments. In the test tube this interaction between actin and myosin is measured as an increase in the rate of ATP hydrolysis (5).

Regulation of phosphorylation. Although smooth muscle and nonmuscle vertebrate cells contain actin, myosin, and tropomyosin, they do not contain troponin. In these cells the major form of regulation appears to directly involve the myosin molecule rather than a complex of proteins residing on the actin filament. Myosin, in smooth muscle and nonmuscle cells can exist in two forms, a phosphorylated form and a nonphosphorylated form. The only difference between these forms is that in one case a phosphate group is covalently (but reversibly) linked to a particular serine residue, located on the $20,000-\mathrm{mol}$ wt light chain of myosin (Fig. I). When both myosin light chains are phosphorylated, actin can increase the rate at which myosin hydrolyzes ATP. When they are not phosphorylated, or when only one light chain is phosphorylated, actin cannot activate myosin, and the ATP hydrolysis rate remains very low $(6,7)$. Phosphorylation of a specific serine residue on the 20,000 -mol wt light chain of myosin apparently removes the inhibition to actin-myosin interaction. This form of regulation appears to pertain to all vertebrate smooth muscle and nonmuscle cells as well as to some invertebrate skeletal muscles such as those found in the telson muscle of the Limulus (8).
Similar to skeletal and cardiac muscle, contractile activity in smooth muscle and nonmuscle cells is initiated by a rise in the calcium concentration from $10^{-7}$ to $10^{-5} \mathrm{M}$. But in smooth and nonmuscle cells calcium binds to the calcium-binding protein, calmodulin, instead of to troponin C. Calmodulin is a relatively lowmolecular-weight (mol wt $=17,000)$ protein that contains four calcium-binding sites. In this property and in many of its other physical and chemical characteristics it resembles troponin C. However, calmodulin, unlike troponin $\mathrm{C}$, binds to and activates the biological activities of a number of different proteins, many of which are enzymes. One of these enzymes is myosin light chain kinase (MLCK), ${ }^{1}$ which catalyzes the phosphorylation of the particular serine residue alluded to above, which is located on the $20,000-\mathrm{mol}$ wt light chain of myosin. This enzyme is totally inactive unless bound to the calcium-calmodulin complex $(9,10)$.

Under certain conditions phosphorylation of myosin may also play an important role in regulating and maintaining myosin filaments. Recent work from a number of laboratories has shown that individual myosin molecules can assume two distinct conformations, folded and extended (11-13). Phosphorylation of myosin favors the extended form of the myosin molecule and it has been suggested that phosphorylation may thus contribute to filament formation. Furthermore, phosphorylation of myosin has been shown to stabilize preexisting myosin filaments in the presence of ATP $(11,14)$.

$M L C K$. The enzyme catalyzing the phosphorylation of myosin is substrate specific in that it only catalyzes

\footnotetext{
${ }^{1}$ Abbreviation used in this paper: MLCK, myosin light chain kinase.
} 
the phosphorylation of myosin light chains. Although MLCK requires the calcium-calmodulin complex for activity, it is itself a substrate for the enzyme cyclic (c)AMP-dependent protein kinase that modulates this activity. As Fig. 1 illustrates, MLCK can be phosphorylated both in the presence and absence of bound calcium-calmodulin complex. When MLCK is phosphorylated by cAMP-dependent protein kinase in the presence of bound calcium-calmodulin, $1 \mathrm{~mol}$ of phosphate is incorporated into MLCK with no apparent effect on MLCK activity. On the other hand, phosphorylation of MLCK when calmodulin is not bound results in a more rapid rate of incorporation of phosphate and leads to a total of $2 \mathrm{~mol}$ of phosphate being incorporated. The enzyme with two bound phosphates per molecule is less active than the mono- or unphosphorylated enzyme (15). The decrease in activity reflects a decreased affinity of the diphosphorylated enzyme for calmodulin and hence suggests some kind of interaction between the calmodulin-binding site and the phosphorylated sites.

The phosphorylation of myosin kinase by cAMPdependent protein kinase may help to explain the effects of certain hormones on the contractile activity of smooth muscles and nonmuscle cells. Unlike skeletal and cardiac muscle, a rise in cAMP in smooth muscles and nonmuscle cells is often associated with a decrease, rather than an increase, in contractile activity. The relaxation of bronchial smooth muscles following the administration of norepinephrine is an example of this phenomenon. Similarly, treatment of blood platelets with drugs that raise intracellular cAMP results in the inhibition of platelet aggregation and secretion. In both cases it is possible that the mechanism for the decrease in contractile activity might involve the interaction of the hormone with the beta adrenergic receptor on the cell surface, activation of adenylate cyclase with increased production of cAMP, the consequent activation of cAMP-dependent protein kinase and the phosphorylation of MLCK. Since phosphorylation of MLCK decreases its activity, the activity of the phosphatase(s) catalyzing dephosphorylation of smooth muscle myosin would be dominant, and phosphorylated (or activated) myosin would be converted to the dephosphorylated form. The importance of this mechanism for relaxing smooth muscles is not yet established at present, and data for (16) and against (17) its role in modulating smooth muscle relaxation has been published. Recent data show that treatment of intact strips of tracheal smooth muscle with forskolin, a drug known to increase cAMP levels, results in increased phosphorylation of MLCK, both before and after treatment of the muscle with the contractile agonist methacholine (18). In interpreting such experiments, it is important to bear in mind that activation of cAMP-dependent protein kinase may lead to phosphorylation of a number of proteins, including those involved in controlling the intracellular concentration of calcium.

Other forms of regulation. Other forms of regulation play an important role in modulating the contractile activity of smooth muscle and nonmuscle cells. In contrast to muscle cells, actin in nonmuscle cells exists in at least two forms, filamentous and oligomeric. Formation, cleavage, and modification of actin filaments is one way in which contractile activity can be modified and a number of proteins capable of regulating actinfilament formation have been described $(2,19)$.

In smooth muscle cells at least two laboratories have suggested additional forms of regulation involving a separate set of regulatory proteins that interact with actin. One of these is similar in many respects to the troponin-tropomyosin system present in striated muscle and is independent of phosphorylation (20). The second involves a calmodulin-binding protein, caldesmon, and may work to modify the effects of phosphorylation by myosin kinase (21). Presently neither of these systems has been defined to the same extent as myosin phosphorylation, and the prevailing opinion favors myosin phosphorylation as the dominant regulatory system in smooth muscle contractile activity. The possibility that smooth muscle myosin might serve as substrate for protein kinases, other than MLCK, has been raised following recent experiments with epidermal growth factor (22). The growth factor activates a kinase that catalyzes phosphorylation of two tyrosine residues in the isolated light chains of smooth muscle myosin. The calcium-activated phospholipid-dependent kinase, protein kinase $C(23)$, also catalyzes the phosphorylation of myosin on the $20,000-\mathrm{mol}$ wt light chain, but at a serine site that is different than that phosphorylated by MLCK. Interestingly, phosphorylation of myosin by protein kinase $\mathrm{C}$, following phosphorylation by MLCK, results in incorporation of phosphate into both sites and decreases the actin-activated myosin Mg ATPase activity.

Phosphatases. Although a great deal is known about MLCK, relatively little is known about the enzymes responsible for dephosphorylation of myosin and MLCK. One phosphatase that has been purified to apparent homogeneity from turkey gizzard smooth muscle has been shown to be composed of three different subunits (24). The holoenzyme $(165,000 \mathrm{~mol} \mathrm{wt})$ is capable of dephosphorylating MLCK, and thereby restoring the ability of MLCK to bind calmodulin with high affinity, thereby restoring its activity. The holoenzyme does not dephosphorylate myosin, but when it is dissociated into its catalytic subunit $(38,000 \mathrm{~mol} \mathrm{wt})$, it is capable of catalyzing this reaction.

Summary. The reversible phosphorylation of myo- 
sin appears to play an important role in regulating the contractile activity of smooth muscle and nonmuscle cells. Phosphorylation of myosin has been described in skeletal and cardiac muscle, but in these cells its function appears to be markedly different from that found for smooth muscle and nonmuscle cells. In striated muscles, phosporylation does not play a primary role in regulating contractile activity, but appears to modulate the contractile response by decreasing ATP utilization $(25,26)$.

The major kinase catalyzing phosphorylation of myosin in smooth muscle and nonmuscle cells is a calcium-calmodulin dependent enzyme, MLCK, which itself can be regulated by phosphorylation by cAMPdependent protein kinase $(15,27)$. Recent experiments have suggested that both myosin and MLCK might also be substrates for additional kinases. The significance of these phosphorylations and how they might act to regulate smooth muscle and nonmuscle contractile activity should become evident in the next few years. Understanding how contractile activity is regulated, in turn, could aid efforts to decipher numerous pathological processes such as coronary artery spasm, defects in cell secretion, and uncontrolled cell division.

\section{ACKNOWLEDGMENTS}

The author wishes to thank those investigators who permitted reference to their work prior to publication. He also thanks Ms. Holly R. Chernin for her fine editorial assistance and Dr. James R. Sellers for reading the manuscript.

\section{REFERENCES}

1. Adelstein, R. S., and E. Eisenberg. 1980. Annu. Rev. Biochem. 49:921-956.

2. Lind, S. E., and T. P. Stossel. 1982. Prog. Hemostasis Thromb. 6:63-84.

3. Huxley, A. F., and R. Niedergerke. 1954. Nature (Lond.). 173:971-973.
4. Huxley, H. E., and J. Hanson. 1954. Nature (Lond.). 173:973-976.

5. Chalovich, J., P. B. Chock, and E. Eisenberg. 1981. J. Biol. Chem. 256:575-578.

6. Sellers, J. R., M. D. Pato, and R. S. Adelstein. 1981. J. Biol. Chem. 256:13137-13142.

7. Persechini, A., and D. J. Hartshorne. 1981. Science (Wash. DC). 213:1383-1385.

8. Sellers, J. R. 1981. J. Biol. Chem. 256:9274-9278.

9. Dabrowska, R., D. Aromatorio, J. M. F. Sherry, and D. J. Hartshorne. 1977. Biochem. Biophys. Res. Commun. 78:1263-1272.

10. Adelstein, R. S., and C. B. Klee. 1981. J. Biol. Chem. 256:7501-7509.

11. Suzuki, H., H. Onishi, K. Takahashi, and S. Watanabe. 1978. J. Biochem. 84:1529-1542.

12. Trybus, K. M., T. W. Huiatt, and S. Lowey. 1982. Proc. Natl. Acad. Sci. USA. 79:6151-6155.

13. Craig, R., R. Smith, and J. Kendrick-Jones. 1983. Nature (Lond.). 302:436-439.

14. Scholey, J. M., K. A. Taylor, and J. Kendrick-Jones. 1980. Nature (Lond.). 287:233-235.

15. Conti, M. A., and R. S. Adelstein. 1981. J. Biol. Chem. 256:3178-3181.

16. Kerrick, W. G. L., and P. E. Hoar. 1981. Nature (Lond.). 292:253-255.

17. Miller, J. R., P. J. Silver, and J. T. Stull. 1983. Mol. Pharmacol. In press.

18. de Lanerolle, P., M. Nishikawa, and R. S. Adelstein. 1983. Biophys. J. 41:370a. (Abstr.).

19. Korn, E. D. 1982. Physiol. Rev. 62:672-737.

20. Mikawa, T., Y. Nonomura, M. Hirata, S. Ebashi, and S. Kakiuchi. 1978. J. Biochem. 84:1633-1636.

21. Sobue, K., Y. Muramoto, M. Fujita, and S. Kakiuchi. 1981. Proc. Natl. Acad. Sci. USA. 78:5652-5655.

22. Gallis, B., A. M. Edelman, J. E. Casnellie, and E. G. Krebs. 1983. J. Biol. Chem. In press.

23. Nishikawa, M., H. Hidaka, and R. S. Adelstein. 1983. J. Biol. Chem. In press.

24. Pato, M. D., and R. S. Adelstein. 1983. J. Biol. Chem. 258:7047-7054.

25. Crow, M. T., and M. J. Kushmerick. 1982. Science (Wash. DC). 217:835-837.

26. Cooke, R., K. Franks, and J. T. Stull. 1982. FEBS (Fed. Eur. Biochem. Soc.) Lett. 144:33-37.

27. Hathaway, D. R., C. R. Eaton, and R. S. Adelstein. 1980 Nature (Lond.). 291:252-254. 